\title{
A NEW COORDINATE FOR PELAGIC AVIAN NAVIGATION
}

D C W Morley

Tushino Ltd, 2 Church Park, Lea Town, Preston PR4 0SJ

http://www.tushino.co.uk

dcw.morley@physics.org

\section{ABSTRACT}

The problem of devising a navigation scheme for ocean dwelling birds has been that there just do not seem to be enough candidates for coordinates. Here it is shown that a gravitational measurement can yield an approximate latitude. This matter being accepted, much of the navigation problem then resolves itself.

\section{INTRODUCTION}

A one kilogram weight placed on the earth surface experiences gravitational forces. There are two main components : The "Earth-g" which is constant and measures $9.81 \mathrm{~m} / \mathrm{s}^{2}$, and the "Astronomical-g" which is a much smaller force and lies in the range of a few hundred microGals. The Earth-g is constant but the Astronomical-g, which reflects the movement of the rotating earth past the sun and the moon, varies in a roughly sinusoidal fashion with a period between maxima of a little over 24 hours. Calculation, reproduced in outline here, shows that the difference between the maxima and minima of the Astronomical-g is related to latitude.

\section{UNITS}

It is practice to measure gravitation forces in Gals.

$1 \mathrm{Gal}$, the unit used for gravity surveys, is defined as an acceleration of $1 \mathrm{~cm} / \mathrm{s}^{2}$ or $10^{-02} \mathrm{~m} / \mathrm{s}^{2}$ or $10^{+07} \mathrm{~nm} / \mathrm{s}^{2}$ Thus $\mathrm{g}=981 \mathrm{gals} 1 \mathrm{microGal}=10 \mathrm{~nm} / \mathrm{s}^{2}$ or $10^{-08} \mathrm{~m} / \mathrm{s}^{2}$

\section{OUTLINED CALCULATION}

Bearing in mind that the Astronomical-g is the driving force behind oceanic tides, and the lesser known earth tides, we can go first to the well studied paper on the matter, that by I M Longman.(Ref A) The calculation is of some complexity because the earth rotates in one plane which is angled to the (ecliptic) plane in which the Earth rotates around the sun. The moon rotates in yet another plane. The geometry becomes less than straightforward.

Fortunately, a Python package is available from GitHub which enables numbers to be easily derived from Longman's work and allows the universal time to be entered.(Ref B). I have placed a user friendly embodiment of this at a web site (Ref C). In this embodiment, the date and time can be entered, the latitude and longitude of the test point and its altitude. As we have in mind sea birds, the altitude term in Astronomical-g can usually be set to zero but, as will be discussed in Ref $C$, not an implied altitude term in Earth-g.

By running the routine the value of Astronomical-g can be found as a function of time, gmax and gmin determined and finally $\mathrm{dg}$.

Table A shows the values in microGals for different latitudes in March 2015

\begin{tabular}{|l|l|l|l|}
\hline lat & gmin & gmax & dg \\
\hline 0 & -95 & 201 & 296 \\
\hline 10 & -96 & 196 & 292 \\
\hline 20 & -96 & 178 & 274 \\
\hline 30 & -97 & 147 & 244 \\
\hline 40 & -99 & 106 & 205 \\
\hline 50 & -103 & 63 & 166 \\
\hline 60 & -106 & 21 & 127 \\
\hline 65 & -106 & 8 & 107 \\
\hline 70 & -106 & -17 & 89 \\
\hline 80 & -106 & -49 & 57 \\
\hline
\end{tabular}


The curve moves a little as you go through the year. Table B shows the values for different latitudes in September 2015

\begin{tabular}{|l|l|l|l|}
\hline lat & $\min g$ & $\max g$ & $\mathrm{dg}$ \\
\hline 0 & -82 & 166 & 248 \\
\hline 10 & -83 & 163 & 246 \\
\hline 20 & -83 & 144 & 227 \\
\hline 30 & -83 & 113 & 186 \\
\hline 40 & -83 & 74 & 157 \\
\hline 50 & -85 & 39 & 124 \\
\hline 60 & -83 & 4 & 87 \\
\hline 65 & -85 & -13 & 72 \\
\hline 70 & -86 & -30 & 56 \\
\hline 80 & -83 & -56 & 27 \\
\hline
\end{tabular}

These curves are shown in the figure. (The upper curve is for March)

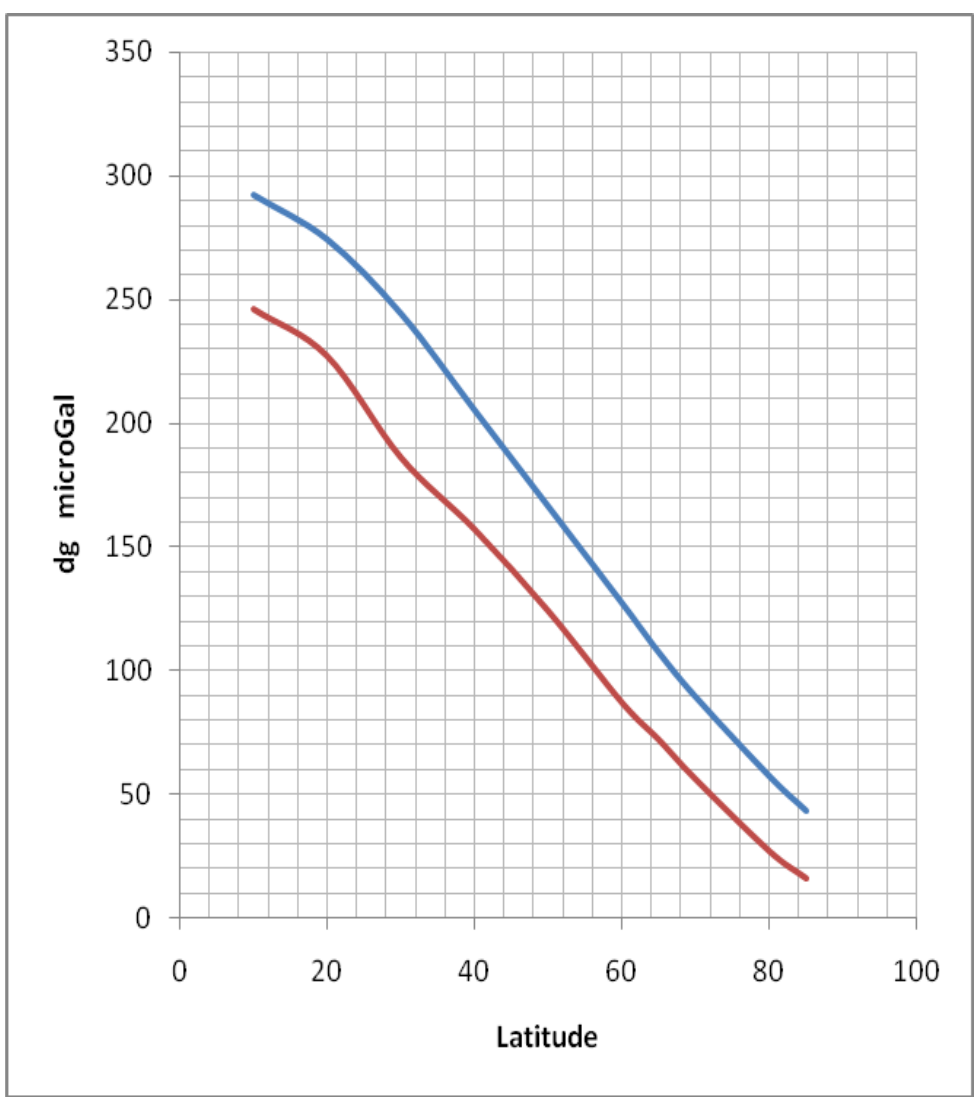




\section{SIGNIFICANCE FOR PELAGIC NAVIGATION - THE "DGF METHOD"}

What is needed to establish a position over an extended and featureless ocean are two independent, detectable and measurable quantities whose isolines intersect. The isolines do not need to be orthogonal in the manner of latitude and longitude.

If it is assumed that birds can measure (1) the total magnetic field $\mathrm{F}$ measured in nanoteslas and (2) derive a latitude from dg then most of the navigation becomes simple. Indeed in some cases it becomes almost trivial. Maps of the total magnetic field can be seen at Ref $D$.

In the proposed scheme dg will play a role similar to latitude. The total magnetic field $\mathrm{F}$ will play the role simply of the second coordinate and it shouldn't be thought of as "longitude by another name". Looking at a magnetic field map (Ref $D$ ) will quickly quash that idea.

Very many birds nest or roost or gather to feed around the limits of the Antarctic continent. A bird can find any position on the shore line or on a closely offshore island simply by following a line of $F$. This can immediately be seen by looking at Ref D and finding the Polar Stereographic Antarctic Main Field F. In this case $\mathrm{F}$ is one coordinate and the easily visible shore line is the other.

Similarly, nesting sites along many continental shores can be reached just by following a line of constant $F$.

Effectively, any substantially extended shore line is a free-issue third co-ordinate

The problem of finding an island is a little more complicated in that the bird will follow a latitude line by measuring dg and watch $\mathrm{F}$ until the two desired values overlap. $\mathrm{dg}$ is a first coordinate and $\mathrm{F}$ is a second.

At this location which will only be approximate the bird must resort to secondary methods. I have put something about this in the supplementary paper $\mathrm{C}$ in Ref $\mathrm{C}$

\section{MEASURABILITY}

It is known that many birds can detect the earth's magnetic field in some sense or another Refs $E$ and $F$. Beason Ref $\mathrm{G}$ and Winklhofer Ref $\mathrm{H}$ have reviewed the possible sensitivities of birds to changes in $\mathrm{F}$ and in some birds the suggested sensitivity seems to be compatible with that needed for ocean navigation.

$\mathrm{F}$ is a scalar and does not depend on the attitude of the bird in flight as would, say, magnetic inclination, and is therefore the preferred candidate for an animal which continually changes attitude and direction.

I do not know of any direct experimental evidence that birds can measure Astronomical-g. The matter has not come under scrutiny until now.

There is, however, in marine biology and terrestrial botany, a strong stream of belief that shellfish and plants have their behaviour synchronised to the lunar cycle. So far as I am aware there is not a definitive experiment that links behaviour to changes in $\mathrm{g}$. There always seems to have been a possible loop hole in the experiments. One of the hindrances to the full evaluation of these studies might have been the cost of sufficiently sensitive gravimeters.

There are some further hindrances to measurement and I have discussed these in the downloadable document with the calculational tools Ref $C$

\section{REFERENCES}

(A) I.M. Longman Journal of Geophysical research 64 (12) pp2351-2355 (1959)

(B) https://pypi.org/project/tidegravity Zachery P. Brady and John R. Leeman

(C) http://www.3ccc3.com

(D) https://www.ngdc.noaa.gov/geomag/WMM/image.shtml 
(E) See for example Wiltschko R and Wiltschko W "Magnetoreception in Birds" J Roy. Soc. Interface Vol 16 20190295 http://dx.doi.org/10.1098/rsf.2019.0295 and

(F) W W Cochran, H Mouritsen \& M Wikelski "Migrating songbirds..." Science 304 pp405-408 (2004)

(G) R C Beason "Mechanisms of Magnetic Orientation in Birds" Integ. Comp. Biol. 45 pp565-573 (2005)

(H) M Winklhofer "The Physics of Geomagnetic-Field Transduction in Animals" IEEE Trans on Magnetics 45 p 5260 (2009)

Acknowledgement : I am indebted to Mr James Docherty for assistance with coding. 